Planetary Systems in the Universe - Observation, Formation and Evolution

Proceedings IAU Symposium No. 202, (C)2004 IAU

Alan Penny, Pawel Artymowicz, Anne-Marie Lagrange, \& Sara Russell, eds.

\title{
Reversing type II migration: resonance trapping of a lighter giant protoplanet
}

\author{
F.S. Masset and M.D. Snellgrove \\ Astronomy Unit, School of Mathematical Sciences, Queen Mary 86 \\ Westfield College, 327 Mile End Road, London E1 4NS, UK
}

\begin{abstract}
We present new results related to the coupled evolution of a two giant planet system embedded in a protoplanetary disk, in which a Saturn mass protoplanet is trapped in an outer mean motion resonance with a Jupiter mass protoplanet. The gaps opened in the disk by the two planets overlap, therefore the two planet system exchanges angular momentum with the disk at the inner planet's Inner Lindblad Resonances (ILRs) and at the outer planet Outer Lindblad Resonances (OLRs). Since the torques are proportional to the square of the planet masses, and since the inner planet is about three times more massive than the outer one, the ILR torques are favored by a factor $\sim 10$ with respect to the one planet case. In the case presented here, this leads to a positive differential Lindblad torque and consequently an outwards migration. We briefly discuss the long-term behavior of the system, which could account for the high eccentricities of the extra-solar planets with semi-major axis $a>0.2 \mathrm{AU}$.
\end{abstract}

\section{Introduction}

We consider the coupled evolution of a system of giant protoplanets embedded in a minimum mass protoplanetary disk, consisting of two non-accreting cores with masses $1 M_{J}$ and $0.29 M_{J}$ (where $M_{J}$ is one Jovian mass), which we call respectively "Jupiter" and "Saturn". This problem is addressed through numerical simulations. After a brief description of the codes we have used, we present the results of a typical run and discuss several issues.

\section{Numerical codes description and initial setup}

In order to investigate the long-term behavior of the embedded Jupiter and Saturn system, we used two independent fixed Eulerian grid based hydrocodes: NIRVANA (Ziegler \& Yorke, 1997) and FARGO (Masset, 2000). They gave very similar results. They consist of a pure N-body kernel embedded in a hydrocode which provides a tidal interaction with a $2 \mathrm{D}$ non self-gravitating gaseous disk. In the following our length unit is $5.2 \mathrm{AU}$, the mass unit is one solar mass, and the time unit is the initial orbital period of Jupiter. In the run presented here the grid resolution adopted is $N_{r}=122$ and $N_{\theta}=300$ with a geometric spacing of the interzone radii. The grid outer boundary is at $R_{\max }=5$ and its inner boundary is at $R_{\min }=0.4$. The protocores start their evolution with semi-major axis respectively $a_{j}=1$ and $a_{s}=2$. The disk surface density is uniform and corresponds to two Jupiter masses inside Jupiter's orbit. The effective viscosity 

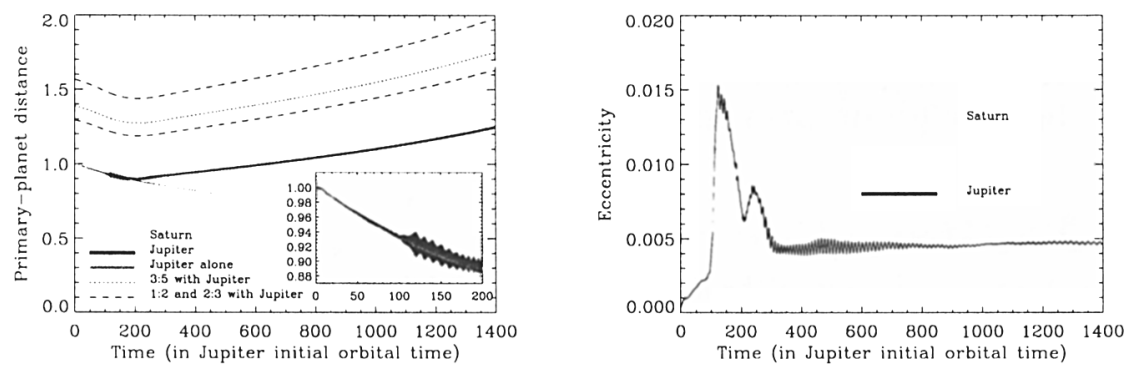

Figure 1. Primary-planet distances and planet eccentricities as a function of time. On the left plot the medium grey curve shows the results of a Jupiter only test run.

$\nu$, the nature of which remains unclear and is usually thought to be due to turbulence generated by MHD instabilities (Balbus \& Hawley 1991), is assumed to be uniform through the disk and corresponds to a value of $\alpha \simeq 6 \cdot 10^{-3}$ in the vicinity of Jupiter's orbit. The disk aspect ratio is uniform and constant and set to $H / r=0.04$.

\section{Run results}

The results are presented in Fig. 1. The mass of Jupiter is sufficient to open a deep gap and hence it settles initially in a type II migration (Nelson et al. 2000) and behaves as if it was the only planet in the disk (see test run), whereas Saturn is unable to fully empty its co-orbital region, and therefore starts a much faster migration. It reaches the 1:2 resonance with Jupiter at time $t \simeq 110$. The planets then get higher eccentricities, and Saturn's migration rate is reduced. Saturn's eccentricity increases again rapidly as it passes through the 3:5 resonance with Jupiter at $t \simeq 220$, and eventually it gets trapped into the $2: 3$ resonance. Both planets then steadily migrate outwards.

\subsection{Interpretation}

We define the system of interest as the system composed of the two planets. This resonance locked system interacts with the inner disk through torques proportional to $M_{J}^{2}$, at Jupiter's inner Lindblad resonances (ILR), whereas it interacts with the outer disk through torques proportional to $M_{S}^{2}$ at Saturn's outer Lindblad resonances (OLR). Now Saturn's ILR fall in Jupiter's gap and Jupiter's OLR fall in Saturn's gap so their effect is weakened compared to the situation where Jupiter is alone. As $M_{J}^{2} / M_{S}^{2} \sim 10$, the torque imbalance does not favor an inwards migration as strongly as in a one planet case, and can even lead to a positive differential Lindblad torque on the two planet system. Actually one can estimate what the maximum mass ratio of the outer planet to the inner one should be to get a migration reversal, if one neglects the Inner Lindblad torque on the outer planet and the Outer Lindblad torque on the inner planet. One 
can show, with conservative assumptions, that the reversal occurs if $\mu_{S} / \mu_{J}<q_{c}$, with $q_{c} \sim 0.6$ (Masset \& Snellgrove 2000).

\section{Discussion}

We have performed a series of "restart runs" in order to check for a variety of behaviors. These runs are restarted once the planets are locked in resonance, and only one parameter is changed at one time. These runs have enabled us to: (i) Confirm that the differential Lindblad torque on the system is positive, with a restart run in which the aspect ratio is changed (see Masset \& Snellgrove 2000 for further details).

(ii) Check that the accretion onto Jupiter does not change the outwards migration rate, except in a maximally accreting case.

(iii) Check that the situation presented here is a steady state one. Indeed, the common gap turns out to be 'permeable' to the flow of material from the outer disk to the inner one, since in all our runs the mass flow through the gap adjusts to $3 \pi \nu \Sigma_{0}+2 \pi r_{s} \dot{r}_{s} \Sigma_{0}$, where $\Sigma_{0}$ is the disk unperturbed surface density and $r_{s}$ is the radius of the separatrix between the last circulating streamline in the outer disk and Saturn's co-orbital horseshoe region.

Since the situation presented here is a steady state one, the outwards migration can be sustained as long as the planets are trapped into resonance. This is not necessarily the case: it will be presented elsewhere in greater detail how Saturn can be moved apart from the $2: 3$ resonance with Jupiter and be sent further out in the disk on a short timescale (a few tens of orbits). On the other hand, it should be noted that if the planets are still locked in resonance when the gas disappears, the eccentricities will not be confined anymore to small values by the co-orbital material, and the system is likely to become unstable. This could result in the ejection of one planet, while the other one would remain on an eccentric orbit.

\section{References}

Balbus S.A., \& Hawley J.F. 1991, ApJ, 376, 214

Masset F. 2000, A\&AS, 141, 165

Masset, F., \& Snellgrove, M. 2000, MNRAS, in press

Nelson R.P., Papaloizou J.C.B., Masset F., \& Kley W. 2000, MNRAS, 318, 18

Ziegler U., \& Yorke H.W. 1997, Comp. Phys. Comp., 101, 54 